\title{
Reducing the maximum power consumption of a group of sucker-rod pumps with the mechatronic drive by optimizing the control algorithm
}

\author{
Mikhail Larin*, and Andrey Volkov \\ Peter the Great St. Petersburg Polytechnic University, 29, Polytechnicheskaya, Saint-Petersburg, 195251, Russia
}

\begin{abstract}
For the development of low- and medium-rate wells, the most commonly used installations are sucker-rod pumps. The group of sucker-rod pumps connected to the same power supply system is installed on the wells of one oilfield. The work of a sucker-rod pump is cyclical. Obviously, there is a relationship between the maximum power consumption of a group of cycle drives and the performance of this group. Selecting such a strategy for reducing the maximum power consumption, in which there will be no significant performance reduction, is an urgent problem. In this article, theoretical approaches are formulated for constructing the simulation model of a sucker-rod pump group as a group of cycle drives with unequal cycles. Several group control strategies that reduce the maximum value of power consumption are considered.
\end{abstract}

\section{Introduction}

Today, the state of the oil industry in Russia is characterized by an increase in the number of idle wells. The main reason for the transfer of wells to the category of inactive with subsequent conservation is the low oil flow rate. For the development of low and medium-rate wells, the most commonly used installations are suckerrod pumps [1]. Usually non-balancer sucker-rod pumps are used for the development of low and medium-yield deposits, due to their small weight and size, as well as relatively little time and low economic costs during delivery and installation compared to the balanced sucker-rod pumps type [2].

Due to the absence of a load balancer, this type of sucker-rod pumps has high peak power consumption. The operation of each of the sucker-rod pump units can be considered as the operation of a cycle mechatronic drive with repeated load cycles [3]. In the case of the uncoordinated operation of the sucker-rod pump group, the peak values of the sucker-rod pump power consumption may overlap. That leads to high requirements for the power supply system in terms of rated power [4]. Providing a group of technological equipment with energy in conditions of remoteness from settlements and power lines is a complex issue [5].

Facts mentioned above make actual the problem of reducing the maximum power consumption of the sucker-rod pump group.

Sucker-rod pump installations in the fields are used in groups and the operation of each of them can be considered as the operation of a cycle drive. Consequently, a group of sucker-rod pump located in the same field and connected to the same power supply system can be considered as a system of cycle drives with different cycles [4].

\section{Theoretical description of the model}

As mentioned above, sucker-rod pumps in the oilfields are used by groups, each of which is connected to a common power source. At the same time, the work of each of them for pumping oil is cyclical, which means that the sucker-rod pumps can be considered as a cyclic drive. However, the cycles of all sucker-rod pumps in the group are different. These differences are caused by the depth of the well, its flow rate, the actual parameters of the electric drives and many other factors. Due to the difference in sucker-rod pump cycles, when all suckerrod pumps in the group are running simultaneously in parallel, the power supply of the group experiences an irregular load with high peak power consumption values. In [6], it was shown that the peak power consumption values of the sucker-rod pump group can be significantly reduced at the cost of a slight decrease in the group's performance when the sucker-rod pump group is coordinated in accordance with certain algorithms. When using recuperation, the peak power consumption can be reduced even further.

However, in [6], the mathematical model of the entire sucker-rod pump group had no generic mathematical definition, and the operation of the control algorithm is considered only in relation to a certain form of the power consumption law.

To compile a mathematical model of the sucker-rod pump group as a group of cycle drives with unequal cycles, we introduce the basic concepts and designations that we will use in the future:

\footnotetext{
* Corresponding author: lay9991@mail.ru
} 
1) The system has $n$ of sucker-rod pumps.

2) All sucker-rod pumps are connected to the same power system with the maximum available power $N_{\text {fact }}=$ const of the power system.

3) There may be an electrical recuperation element with the maximum stored amount of energy $E_{\max }$ in the power system.

4) Each sucker-rod pump has a law of energy consumption $N_{i}\left(t, \boldsymbol{\alpha}_{i}\right)$ for the working cycle, where $i=1, \ldots, n$ - sucker-rod pump index; $\boldsymbol{\alpha}_{i}=$ const - vector of sucker-rod pump working cycle parameters $i$ that are set by external conditions; $T_{i}$ - cycle duration for the sucker-rod pump $i$.

5) All sucker-rod pumps can recuperate power to the recuperation element with the same recovery coefficient $k$.

6) Each sucker-rod pump can be started at time moment $t_{i}^{*}$ and stopped after the working cycle at time moment $t_{i}^{*}+T_{i}$.

7) All sucker-rod pumps are connected to the same upper-level control system, that generate control signal $u_{i}$ about start time $t_{i}^{*}$ for the sucker-rod pump $i$; control signal for all of sucker-rod pumps are $\boldsymbol{u}(t)=\left(u_{1}(t), \ldots, u_{n}(t)\right)^{\mathrm{T}}$.

8) The sucker-rod pump $i$ pumps oil volume $v_{i}$ for the single working cycle.

9) The general pumped oil volume for the suckerrod pump group during the time $t$ is $V(t)=\sum_{i=1}^{n}\left(m_{i}(t) \cdot v_{i}\right)$, where $m_{i}(t)-$ count of completed cycles of the sucker-rod pump $i$ during the time $t$; it is obvious that $m_{i}(t)=f\left(u_{i}(t)\right)$, that leads to $V(t)=V(\boldsymbol{u}(t))$.

10) The solution to the optimization problem for this system is to maximize the pumped volume of the entire group $V(\boldsymbol{u}(t))$ with the given available power $N_{\text {fact }}$.

11)The power required for uncoordinated parallel operation of all sucker-rod pumps is $N_{\text {max }}=\sum_{i=1}^{n} \max _{t} N_{i}\left(t, \boldsymbol{\alpha}_{i}\right)$.

The relationships between these values are written in the form of a system of equations and conditions.

$$
\left\{\begin{array}{c}
E_{\text {rec }}(t, \boldsymbol{u}(t))=\int_{0}^{t}\left(N_{\text {fact }}-\sum_{i=0}^{n} N_{i}^{*}\left(u_{i}(t)\right)\right) d t \\
N_{i}^{*}\left(u_{i}(t)\right)=k_{i}^{*}\left(N_{i}^{\prime}\left(u_{i}(t)\right)\right) \cdot N_{i}\left(u_{i}(t)\right) \\
k_{i}^{*}\left(N_{i}^{\prime}\left(u_{i}(t)\right)\right)=k+(1-k) h\left(N_{i}^{\prime}\left(u_{i}(t)\right)\right) \\
N_{i}\left(u_{i}(t)\right)=N_{i}\left(t-u_{i}(t), \boldsymbol{\alpha}_{i}\right) \cdot s_{i}\left(t, u_{i}(t)\right) \\
s_{i}\left(t, u_{i}(t)\right)=h\left(t-u_{i}(t)\right)-h\left(t-T_{i}-u_{i}(t)\right) \\
\boldsymbol{s}(t, \boldsymbol{u}(t))=\left(s_{1}\left(t, u_{1}(t)\right), \ldots, s_{n}\left(t, u_{n}(t)\right)\right)^{T} \\
h(x) \in\{0,1\}-\operatorname{Heaviside~step~function~}^{n} \\
V(\boldsymbol{u}(t))=\sum_{i=1}^{n}\left(m_{i}(\boldsymbol{u}(t)) \cdot v_{i}\right) \\
t_{i, j+1}^{*} \geq t_{i, j}^{*}+T_{i} \\
E_{\text {rec }}(t, \boldsymbol{u}(t)) \geq 0 \\
E_{\text {rec }} \leq E_{\max } \\
\boldsymbol{u}(t)=\underset{\boldsymbol{u}(t)}{\operatorname{argmax}} \sum_{i=1}^{n}(V(\boldsymbol{u}(t)))
\end{array}\right.
$$

Special attention in this system should be paid to the following conditions:

- $t_{i, j+1}^{*} \geq t_{i, j}^{*}+T_{i}$ - the sucker-rod pump $i$ can start a new working cycle $j+1$ after finishing the current working cycle $j$ only;

- $E_{\text {rec }}(t, \boldsymbol{u}(t)) \geq 0$ is a critical condition of the proper operation of the drive group, since violation of this condition leads to power system overloading;

- $E_{\text {rec }} \leq E_{\max }$ - recuperated amount of energy is limited by the recuperation element capacity. While there is no recuperation element in the power system, the following condition is correct $E_{\text {max }}=E_{\text {rec }}=0$ that leads to the following transformation in the system:

$$
N_{\text {fact }}-\sum_{i=0}^{n} N_{i}^{*}\left(u_{i}(t)\right) \geq 0 .
$$

To solve the optimization problem and study the influence of various control algorithms on the results of the solution, a simulation model was created in the MATLAB program with the $M$ script language. Conditions and dependencies described in the mathematical model of the system (1) are correct and are observed at each moment of the simulated time in the created model. One of the components of this model is a control system that generates control signals for each sucker-rod pump in the group in accordance with the selected algorithm.

\section{Sucker-rod pump group management priority- based algorithm without forecasting}

The main ideas of this algorithm:

- With limitations of the available power $N_{\text {fact }}$ taken into account, the control system must start sucker-rod pumps in a certain sequence in order to meet the critical condition of the existence of the system. 
- Since sucker-rod pumps have different operating cycles of energy consumption $N_{i}\left(t, \boldsymbol{\alpha}_{i}\right)$ and different volumes pumped per cycle $v_{i}$, some of the sucker-rod pumps are more profitable to start than others, in order to maximize the result of the entire system $V(\boldsymbol{u}(t))$. This means that such sucker-rod pumps have a higher priority $p_{i} \in[1, n]$. Priorities are set before starting the system based on the priority indicator $Q_{i}$ calculated according to the selected criterion.

- The decision to start the sucker-rod pump $k$ is made at the current time $t$ without forecasting the possibility that the start of the sucker-rod pump $k$ at the time moment $t$ may prevent the start of a higherpriority sucker-rod pump $l$ that is active in the moment $t^{\prime}$ and will end the movement after some time.

It is necessary to disclose the concept of availability of the sucker-rod pump $k$ for launch. The sucker-rod pump $\mathrm{k}$ is considered available to run when:

- The sucker-rod pump $k$ is not active - this condition makes it possible to start the sucker-rod pump in general, since the active sucker-rod pump cannot be started until the end of the cycle.

$$
\begin{aligned}
& \left\{\begin{array}{c}
s_{k}\left(t^{\prime}, u_{k}\left(t^{\prime}\right)\right)=0 \\
E_{\text {rec }}^{*}\left(t, t^{\prime}\right) \geq 0 \text { при } t \in\left[t^{\prime}, t^{\prime}+T_{k}\right] \\
E_{\text {rec }}\left(t^{\prime}\right)=\int_{0}^{t^{\prime}}\left(N_{\text {fact }}-\left[\sum_{i=0}^{n} N_{i}^{*}\left(u_{i}(t)\right)\right]\right) d t \\
E_{\text {rec }}^{*}\left(t, t^{\prime}\right)=E_{\text {rec }}\left(t^{\prime}\right)-\int_{t^{\prime}}^{t}\left(N_{\text {fact }}-\left[N_{k}\left(t-t^{\prime}\right)+\sum_{i=0}^{n, i \neq k} N_{i}^{*}\left(u_{i}\left(t^{\prime}\right)\right)\right]\right) d t
\end{array}\right. \\
& \left\{\begin{array}{c}
\boldsymbol{p}=\left(p_{1}, \ldots, p_{n}\right)^{T} \\
p_{k}=\min _{i} \boldsymbol{p} \\
s_{k}\left(t^{\prime}, u_{k}\left(t^{\prime}\right)\right)=0 \\
E_{\text {rec } k}^{*}\left(t, t^{\prime}\right) \geq 0 \text { при } t \in\left[t^{\prime}, t^{\prime}+T_{k}\right] \\
E_{\text {rec }}^{*}\left(t, t^{\prime}\right)=E_{\text {rec }}\left(t^{\prime}\right)+N_{\text {fact }} \cdot\left(t^{\prime}-t\right)+\int_{t^{\prime}}^{t}\left[N_{k}\left(t-t^{\prime}\right)+\sum_{i=0}^{n, i \neq k} N_{i}^{*}\left(u_{i}\left(t^{\prime}\right)\right)\right] d t
\end{array}\right. \\
& \left\{\begin{array}{c}
\boldsymbol{Q}=\left(Q_{1}, \ldots, Q_{n}\right)^{T} \\
Q_{k}=\max _{i} \boldsymbol{Q} \\
S_{k}\left(t^{\prime}, u_{k}\left(t^{\prime}\right)\right)=0 \\
E_{\text {rec }}^{*}\left(t, t^{\prime}\right) \geq 0 \text { if } t \in\left[t^{\prime}, t^{\prime}+T_{k}\right] \\
E_{\text {rec }}^{*}\left(t, t^{\prime}\right)=E_{\text {rec }}\left(t^{\prime}\right)+N_{\text {fact }^{\prime}} \cdot\left(t^{\prime}-t\right)+\int_{t^{\prime}}^{t}\left[N_{k}\left(t-t^{\prime}\right)+\sum_{i=0}^{n, i \neq k} N_{i}^{*}\left(u_{i}\left(t^{\prime}\right)\right)\right] d t
\end{array}\right.
\end{aligned}
$$

From the obtained systems, it can be seen that the evaluation of the priority criterion $Q_{i}$ is crucial for this control method. It is reasonable to investigate the behavior of the system with the variants of the priority indicator $Q_{i}$ calculated according to the following criteria:

- the sucker-rod pump performance: $Q_{i}=v_{i}$;
- Starting of the sucker-rod pump $k$ while other sucker-rod pumps will finish its cycles and remain inactive will not lead to a violation of the critical condition for the existence of the system. This system, because when any other And the sucker-rod pump $k$ will be considered as active - if at this time moment $t$ the control system has decided to start the sucker-rod pump $\mathrm{k}$.

The formal formulation of the sucker-rod pump $k$ simplified taking into account the known limits of its calculation: $t \in\left[t^{\prime}, t^{\prime}+T_{k}\right]$. Also, let a higher priority o lower value; this means that the suckerpriority $p_{i}=1$ has the highest priority. are given in the system (3). When through the priority indicator $\boldsymbol{Q}$, the system (4) will be obtained. 
- relative sucker-rod pump performance based on average power consumption per cycle: $Q_{i}=$ $\frac{v_{i}}{N_{\text {i avg }}}$.

\section{Intermediate results}

The simulation model was investigated using a prioritybased control algorithm without forecasting with four variants of determining the priority.

Initial system parameters:

- sucker-rod pump group parameters:

- $n=7$

- $N_{\text {fact }} \in\left[0, N_{\text {max }}\right]$ with step $0.05 N_{\text {max }}$;

- $N_{\text {max }}=1.1219 \cdot 10^{2} \mathrm{\kappa BT}$

$-k_{i}=k=0.3$

- $\boldsymbol{\alpha}_{i}=\left(D_{0}, d_{0}, g, w, a_{f}, r, i, J, H, v_{i}, l_{0 i}, \rho, \rho_{n}, a_{i}\right)^{T}-$ values of the parameters are given in the Table 1 , excepting parameters $v_{i}, l_{0 i} a_{i}-$ values of this parameters are given in the Table 2 . The values are obtained from a uniform distribution with variations $\Delta_{v}= \pm 0.5 v ; \Delta_{l}= \pm 0.5 l_{0} ; \Delta_{a}= \pm 0.1 a$ around the nominal values of parameters accordingly;

- $E_{\text {max }}=\sum_{i=1}^{n} \int_{0}^{T_{i}} N_{i}^{*}(t) \cdot h\left(N_{i}^{*}(t)\right) d t=1.282$. $10^{7} \mathrm{~J}$ - the total energy consumption of suckerrod pump group per one cycle. The value is taken so that the upper limit on the recovered energy does not affect the possibility of starting the sucker-rod pump, if the average recovered power is greater than the average power consumed;

- simulation time $T=\frac{\sum_{i=1}^{n} T_{i}}{n} \cdot 100=785.6 \mathrm{c} \quad$ sufficient time to enter the steady state mode and run out of data in this mode. Further increasing the simulation time increases the model calculation time without significantly affecting the simulation results;

- $t_{\text {step }}=0.1 \mathrm{c}-$ time step between simulation iterations;

- the pumped volume for each of sucker-rod pumps values are given in the Table 2;

- laws of the power consumption during working cycle of sucker-rod pumps $N_{i}\left(t, \boldsymbol{\alpha}_{i}\right)$ are taken in accordance with [7]. Graphs of power consumption laws are shown in Figure 1.

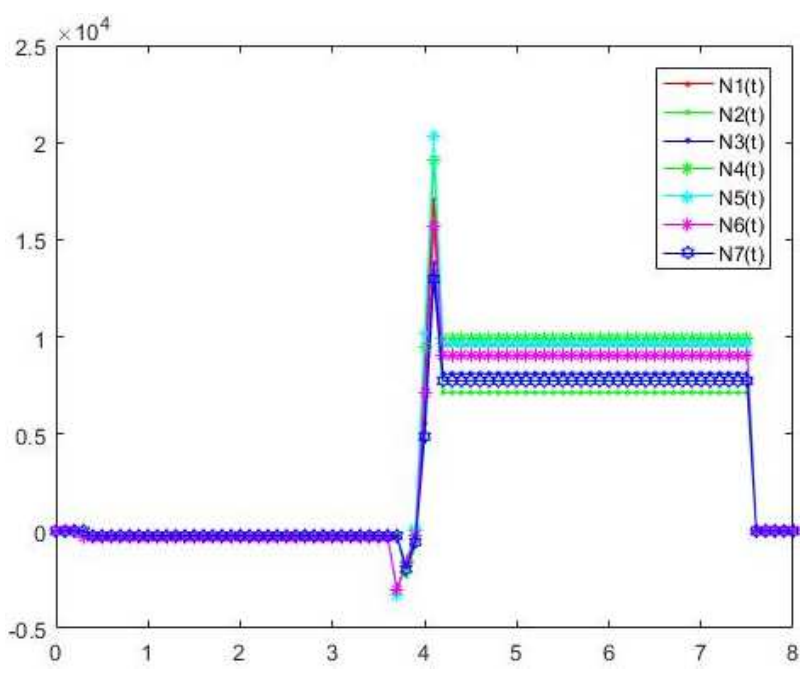

Fig. 1. Graphs of power consumption laws $\boldsymbol{N}_{\boldsymbol{i}}\left(t, \boldsymbol{\alpha}_{\boldsymbol{i}}\right)$ for the sucker-rod pump group

Table 1. Values of elements of the vector of parameters of the sucker-rod pump working cycle

\begin{tabular}{|c|c|}
\hline $\begin{array}{l}\text { Parameter } \\
\text { name }\end{array}$ & Meaning \\
\hline$D_{0}=0.06$ & Sucker-rod pump piston diameter, $m$ \\
\hline$d_{0}=0.028$ & Average rod diameter, $m$ \\
\hline$g=9.8$ & Free fall acceleration, $\mathrm{m} / \mathrm{s}^{2}$ \\
\hline$w=145$ & Angular velocity of the engine, $s^{-1}$ \\
\hline$a_{f}=0.1$ & Reduced dry friction coefficient \\
\hline$r=0.056$ & Gear dividing radius, $m$ \\
\hline$i=24.75$ & Gear ratio of the gearbox \\
\hline$J=0.1$ & $\begin{array}{l}\text { Moment of inertia of the engine armature, } \\
\qquad \mathrm{kg} \cdot \mathrm{m}^{2}\end{array}$ \\
\hline$H=1.2$ & Stroke value of the rod pump, $m$ \\
\hline$v=8$ & $\begin{array}{l}\text { Average value of the volume of the } \\
\text { pumped oil by sucker-rod pump, } l\end{array}$ \\
\hline$l_{0}=1100$ & Average value of the rod length, $m$ \\
\hline$\rho=7800$ & Steel density, $\mathrm{kg} / \mathrm{m}^{3}$ \\
\hline$\rho_{n}=800$ & Oil density, $\mathrm{kg} / \mathrm{m}^{3}$ \\
\hline$a_{2}=1.5$ & $\begin{array}{c}\text { Acceleration of rail braking when moving } \\
\text { down, } \mathrm{m} / \mathrm{s}^{2}\end{array}$ \\
\hline$a_{3}=1.5$ & $\begin{array}{c}\text { Acceleration of rail acceleration during } \\
\text { lifting, } \mathrm{m} / \mathrm{s}^{2}\end{array}$ \\
\hline
\end{tabular}


Table 2. Variations of values of sucker-rod pump parameters

\begin{tabular}{|c|c|c|c|}
\hline $\boldsymbol{i}$ & $\boldsymbol{v}_{\boldsymbol{i}}, \boldsymbol{l}$ & $\boldsymbol{l}_{\mathbf{0} \boldsymbol{i}}, \boldsymbol{m}$ & $\boldsymbol{a}_{\boldsymbol{i}}, \boldsymbol{m} / \boldsymbol{s}^{\mathbf{2}}$ \\
\hline 1 & 8.6204 & 1198.8 & 1.4495 \\
\hline 2 & 7.9935 & 890.4 & 1.569 \\
\hline 3 & 8.3411 & 1012.2 & 1.4586 \\
\hline 4 & 7.0204 & 1238.2 & 1.5009 \\
\hline 5 & 9.5636 & 1209.5 & 1.5689 \\
\hline 6 & 6.5545 & 1126.0 & 1.4474 \\
\hline 7 & 9.3629 & 966.6 & 1.4631 \\
\hline
\end{tabular}

The obtained values of the priority indicators and the corresponding priorities for each variant of the priority criterion are presented in Table 3.

Table 3. Values of the priority indicators and the corresponding priorities for each variant of the priority criterion

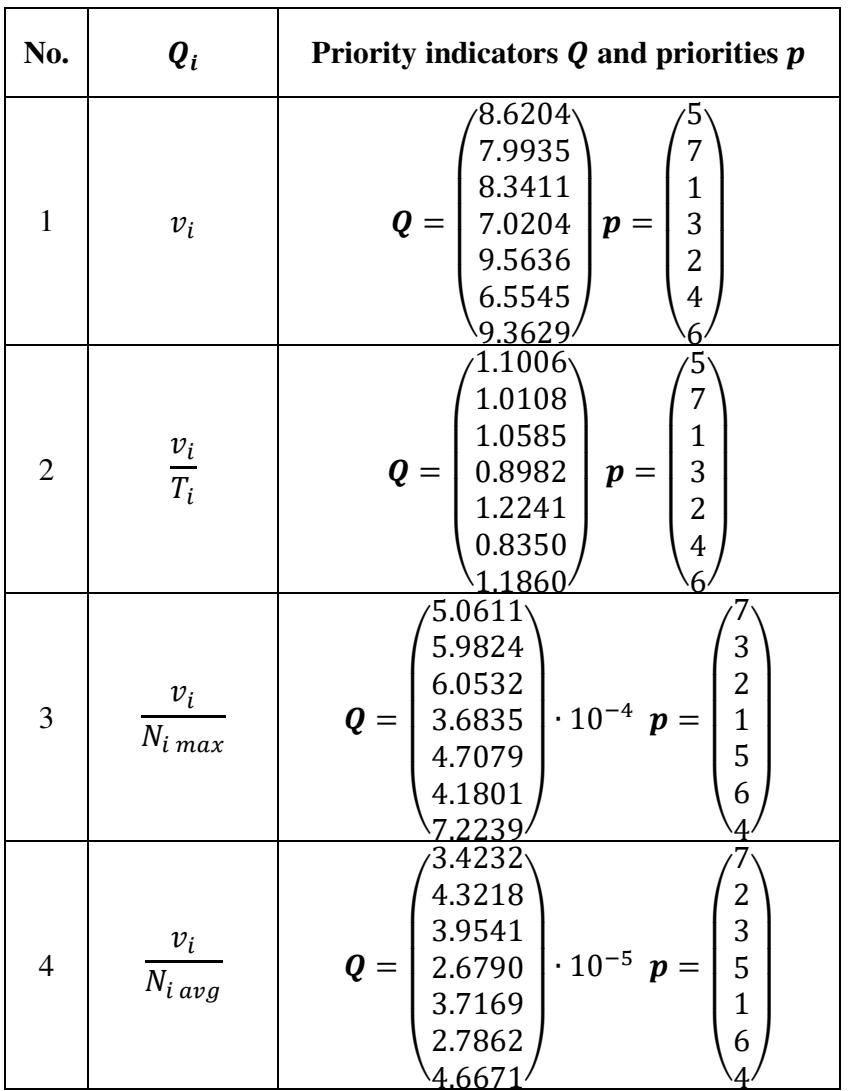

To analyze the results, comparative graphs of the normalized values of $V_{r}=f\left(N_{r}\right)$ are used. Graphs for different variants of prioritization are shown in Figure 2, where $V_{r}(\boldsymbol{u}(t))=\frac{V(\boldsymbol{u}(t))}{V_{\max }(t)}, N_{r}=\frac{N_{\text {fact }}}{N_{\max }}$. From the graphs it can be seen that the algorithm with prioritization by the criterion $Q_{i}=\frac{v_{i}}{N_{i} \text { avg }}$ works for this system better than with others criterions, but other results are also quite close to the obtained maximum result.

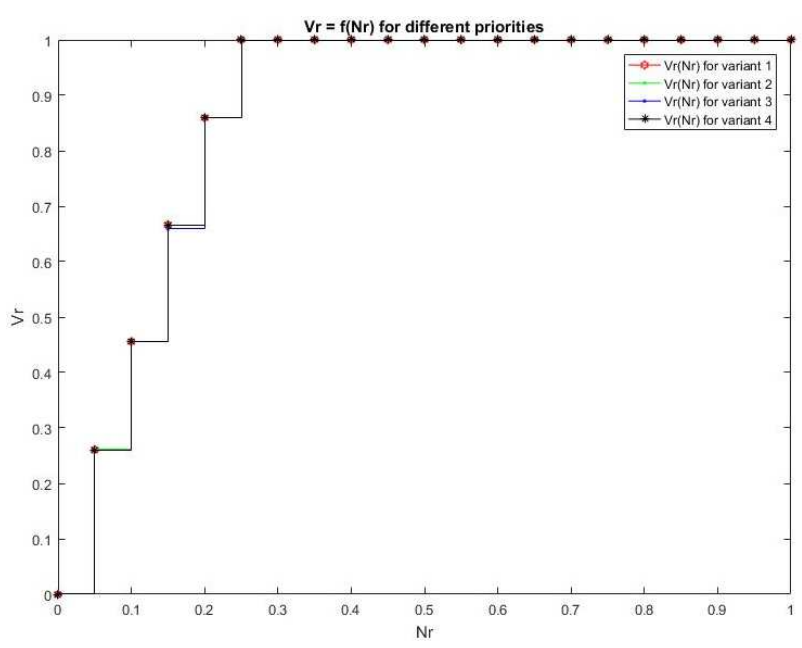

Fig. 2. Graphs of the relative pumped volume of the sucker-rod pumps of group $\boldsymbol{V}_{\boldsymbol{r}}$ function from relative rated power of the power source $\boldsymbol{N}_{\boldsymbol{r}}$ for variants of the prioritization criterion

\section{Application of the simulation model for the sucker-rod pump group with a rectangular power consumption law}

A control algorithm based on priority criteria $Q_{i}=\frac{v_{i}}{N_{i} \text { avg }}$ without forecasting was studied under the rectangular law of energy consumption of the sucker-rod pump at different values of the acceleration duration coefficient $k_{\tau}$. This is necessary to determine the effect of the acceleration duration parameter $k_{\tau}$ to the performance of the sucker-rod pump group, since in the future the acceleration duration coefficient can be set by the control system of the sucker-rod pump group [7]. Initial system parameters:

- sucker-rod pump group parameters:

- $n=9$

- $N_{\text {fact }} \in\left[0, N_{\text {max }}\right]$ with step $0.05 N_{\text {max }}$;

- $N_{\text {max }}=190.04 \mathrm{~kW}$;

- $k_{i}=0, i \in[1, n]$ - this group works without recuperation;

- $\boldsymbol{\alpha}_{i}=\left(k_{i \tau}, A_{i}, T_{i}\right)^{T}$, where $A_{i}\left(\tau_{i}\right)=$ const - work spent on the acceleration of the $\operatorname{rod} ; T_{i}\left(\tau_{i}\right)=$ const - duration of the sucker-rod pump working cycle. $A_{i}$ and $T_{i}$ parameters are given in Table 4;

- $k_{i \tau}=k_{\tau} \in\left[k_{i \tau_{\text {min }}}, 0.5\right]$ with step 0.02;

- where $k_{i \tau_{\min }}=\frac{A}{N_{d r v_{\max } \cdot T_{i}}}, \quad N_{d r v_{\max }}=50 \mathrm{~kW}$;

- $E_{\text {max }}=0$ - this group works without recuperation;

- $\quad$ simulation time $T=\frac{\sum_{i=1}^{n} T_{i}}{n} \cdot 100=988.51 \mathrm{~s}$;

- $v_{i}\left(\tau_{i}\right)=$ const - values are given in Table 4;

- laws of the power consumption during the working cycle of sucker-rod pumps $N_{i}\left(t, \boldsymbol{\alpha}_{i}\right)$ are given in Figure 3: 


$$
\left\{\begin{array}{c}
N_{i}\left(t, \boldsymbol{\alpha}_{i}\right)=\frac{A_{i}}{\tau_{i}} \text { if } t<\tau_{i} \\
N_{i}\left(t, \boldsymbol{\alpha}_{i}\right)=0 \text { if } \tau_{i} \leq t<\left(T_{i}-\tau_{i}\right) \\
N_{i}\left(t, \boldsymbol{\alpha}_{i}\right)=-k_{i} \frac{A_{i}}{\tau_{i}} \text { if }\left(T_{i}-\tau_{i}\right) \leq t<T_{i} \\
\tau_{i}=k_{i \tau} \cdot T_{i}
\end{array}\right.
$$

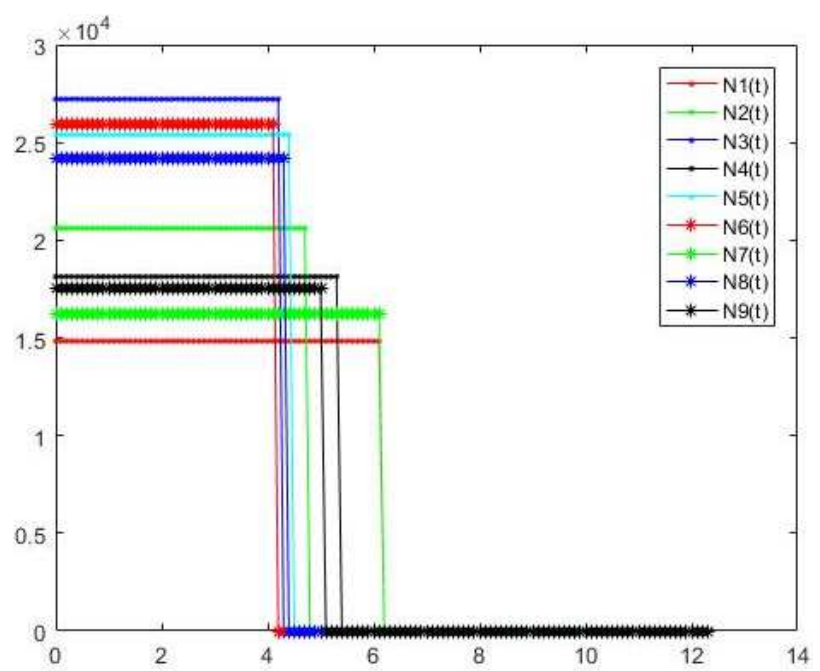

Fig. 3. Sucker-rod pumps power consumption laws $\boldsymbol{N}_{\boldsymbol{i}}\left(\boldsymbol{t}, \boldsymbol{\alpha}_{\boldsymbol{i}}\right)$ during the working cycle with $\boldsymbol{k}_{\boldsymbol{\tau}}=\mathbf{0 . 3 3}$

Table 4. Variations of values of sucker-rod pump parameters

\begin{tabular}{|c|c|c|c|}
\hline $\boldsymbol{i}$ & $\boldsymbol{v}_{\boldsymbol{i}}, \boldsymbol{l}$ & $\boldsymbol{T}_{\boldsymbol{i}}, \boldsymbol{s}$ & $\boldsymbol{A}_{\boldsymbol{i}}, \boldsymbol{k J}$ \\
\hline 1 & 10.974 & 12.251 & 90.86 \\
\hline 2 & 7.672 & 9.408 & 96.94 \\
\hline 3 & 11.328 & 8.434 & 114.76 \\
\hline 4 & 9.949 & 10.732 & 97.28 \\
\hline 5 & 11.047 & 8.880 & 112.73 \\
\hline 6 & 10.899 & 8.313 & 107.75 \\
\hline 7 & 8.095 & 12.299 & 99.92 \\
\hline 8 & 9.202 & 8.619 & 104.26 \\
\hline 9 & 11.256 & 10.030 & 87.75 \\
\hline
\end{tabular}

Dependencies obtained as a result of the simulation are shown in Figure 4. The general nature of the dependencies in the $N_{r}, k_{\tau}, V_{r}$ axes is shown in Figure 5.

The best results were obtained with the acceleration duration coefficient $k_{\tau}=0.5$ while $N_{r} \geq 0.5$. When $N_{r}<0.5$ the best results can be obtained for different values of $k_{\tau}$.

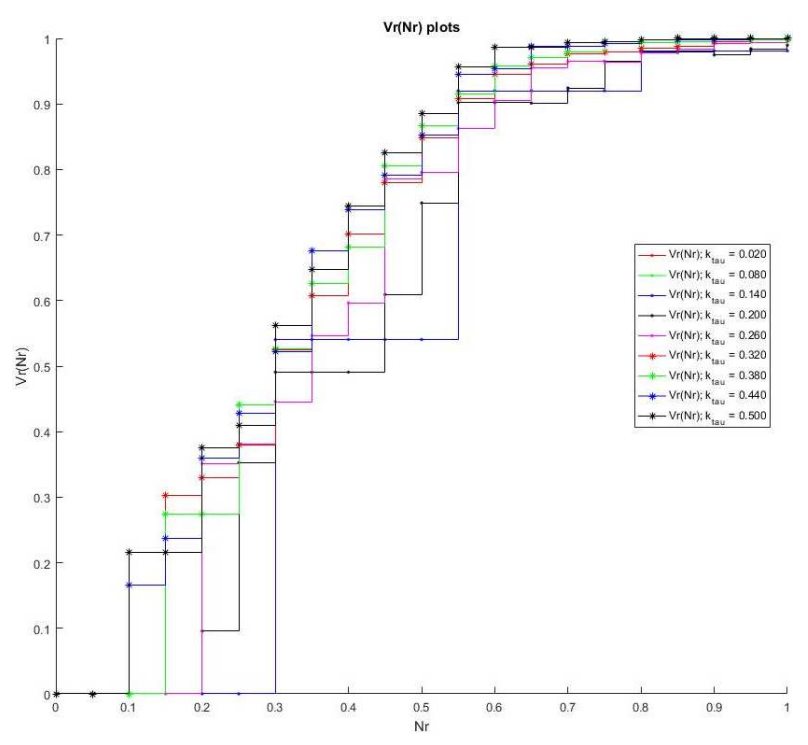

Fig. 4. Dependencies of the relative pumped volume $\boldsymbol{V}_{\boldsymbol{r}}$ of the sucker-rod pumps group on the relative rated power $\boldsymbol{N}_{\boldsymbol{r}}$ of the power supply for different acceleration duration coefficients $\boldsymbol{k}_{\boldsymbol{\tau}}$

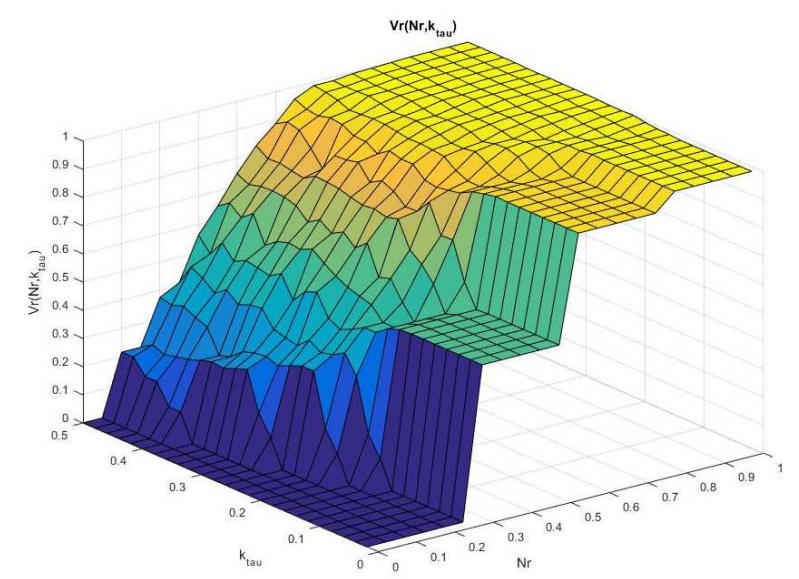

Fig 5. Surface of the relative pumped volume of the sucker-rod pumps of group $\boldsymbol{V}_{\boldsymbol{r}}$ function from the relative rated power $\boldsymbol{N}_{\boldsymbol{r}}$ of the power source and the coefficient of the acceleration duration $\boldsymbol{k}_{\boldsymbol{\tau}}$

\section{Results}

A mathematical model of the sucker-rod pump group with unequal cycles, with an arbitrary form of the law of consumption during the cycle, is obtained. Using this mathematical model, a simulation model was synthesized to solve the problem of optimizing the peak power consumption of the group and to study the influence of various control algorithms on the results of the solution.

The algorithm for controlling the sucker-rod pump group was improved in comparison with the work [6], and the principles of its operation for sucker-rod pump with an arbitrary form of the law of energy consumption in a formal form were formulated.

The resulting simulation model is investigated for a priority-based control algorithm without forecasting for different priority criteria for a group of sucker-rod pumps with a rectangular consumption law. 
A simulation model with a priority-based control algorithm without forecasting for the priority criterion based on the average power consumption was studied with data from the sucker-rod pump group with a rectangular consumption law for various durations and values of active consumption.

The following conclusions can be drawn from the results of the simulation model:

1) For the sucker-rod pump group with $n=7$, the recuperation coefficient $k_{r e c}=0,3$ and the maximum amount of recuperated energy of the system $E_{\max }=1.282 \cdot 10^{7} \mathrm{~J}$ the considered algorithms give similar results. At the same time, a decrease in the relative rated power of the system power supply from the maximum required $N_{r}$ to $25 \%$ does not lead to a loss of group performance; a decrease in $N_{r}$ to $20 \%$ leads to a decrease in performance by $13 \%$.

2) The sucker-rod pump group with $n=9, k_{\text {rec }}=0$ and $E_{\max }=0$ shows the best results with a coefficient of acceleration duration $k_{\tau}=0,5$ while $N_{r} \geq 0,5$. The results obtained indicate that an increase in the acceleration time and a decrease in the power consumption value with a uniform power consumption of each sucker-rod pump during acceleration is preferable in terms of reducing the peak power consumption for the entire sucker-rod pump group. When $N_{r}<0.5$ the best results can be obtained for different values of $k_{\tau}$ that is a separate question for the research. The tendency of increasing the value of $V_{r}$ with increasing $k_{\tau}$ is established.

\section{Acknowledgments}

The reported study was funded by RFBR, project number 20-38-90130.

\section{References}

1. V. V. Andreev, K. R. Urazakov, et al. Handbook of oil production (Nedra Business Center LLC, 2000)

2. M. I. Khakimyanov, Vestnik UGATU 18, 54-60 (2014)

3. F. A. Gizatullin, M. I. Khakimyanov, Electrotechnical and Information Complexes and Systems. 13, 11-18 (2017)

4. E.Camponogara, M.D. Oliveira, M.A.S. De Aguiar, European Journal of Operational Research, 247, 945-956 (2015)

5. M. Yu.Larin, O. N. Matsko, M. N. Polishchuk, M. V. Anisimova, D. V. Moiseev, Materials of the International scientific and technical conference. Sevastopol State University, 45-46, (2016)

6. M. Yu. Larin, T. V. Andreeva, S. O. Pozhidaev, SPbPU Science Week: proceedings of the scientific conference with international participation, 60-62 (2017)

7. A. N. Volkov, O. N. Matsko, A.V. Mosalova, St. Petersburg polytechnic university journal of engineering science and technology. 24, 141-149 (2018) 